\title{
Automatic Identification of Cloud Cover REGIONS USING SURF
}

\author{
Dr. Mohamed Mansoor Roomi ${ }^{\# 1}$ R.Bhargavi ${ }^{\# 2}$ T.M.Hajira Rahima Banu ${ }^{\# 3}$ \\ Department of Electronics and Communication Engineering, Thiagarajar College of \\ Engineering, Madurai \\ 1 smmroomietce.edu \\ 2 r \\ bhargavi22@gmail.com \\ ${ }^{3}$ tmhrbanuegmail. com
}

\begin{abstract}
Weather forecasting has become an indispensable application to predict the state of the atmosphere for a future time based on cloud cover identification. But it generally needs the experience of a well-trained meteorologist. In this paper, a novel method is proposed for automatic cloud cover estimation, typical to Indian Territory Speeded Up Robust Feature Transform(SURF) is applied on the satellite images to obtain the affine corrected images. The extracted cloud regions from the affine corrected images based on Otsu threshold are superimposed on the artistic grids representing latitude and longitude over India. The segmented cloud and grid composition drive a look up table mechanism to identify the cloud cover regions. Owing to its simplicity, the proposed method processes the test images faster and provides accurate segmentation for cloud cover regions.
\end{abstract}

\section{KEYWORDS}

Weather Forecast, SURF, Affine, Threshold.

\section{INTRODUCTION}

Weather is a continuous, data-intensive, multidimensional, dynamic and chaotic process, and these properties make weather forecasting a formidable challenge. There are various techniques involved in weather casting, from relatively simple observation of the sky to highly complex computerized mathematical models. Cloud, an element of weather plays vital role in weather forecasting. Thickening of cloud cover or the invasion of a higher cloud deck is indicative of rain in the near future. Generally, empirical and dynamic approaches are used to forecast weather. The first approach is based upon the occurrence of analogs and is often referred to by meteorologists as analog forecasting. This approach is useful for predicting local-scale weather if recorded cases are plenty. The second approach is based upon forward simulations of the atmosphere, and is often referred to as computer modelling. Because of the grid coarseness, the dynamical approach is only useful for modelling large-scale weather phenomena and may not predict short-term weather efficiently. Most weather prediction systems use a combination of empirical and dynamical techniques. Identification and observation of clouds can provide clues to the severity of approaching bad weather. Current observation methods depend on changes in barometric pressure, weather conditions and sky condition. Human input is still required to pick the best possible forecast model and identify the cloud cover region, which involves pattern recognition, 
teleconnections and knowledge of model performance. The solution for cloud cover identification without human interpretation can be achieved using cloud segmentation as initial process. In [6], a review of cloud classification techniques is provided. Lim etal has proposed Cloud field segmentation via multiscale convexity analysis [5].A multispectral classification of meteorological satellite images using a pixel-clustering algorithm is described in [8]. A number of cloud segmentation approaches have used pixel-wise neural-network-based classifiers that exploit textural and spectral features [7][10-13]. Wenlong etal proposed a Satellite Cloud Image Segmentation Based on the Improved Normalized Cuts Model [17] .In contrast, Dipti.et al [14] have developed an area-based, shape-preserving morphological segmentation scheme for cloud extraction. It is a major concern to identify any trends for weather parameters to deviate from its periodicity, like Natural calamities which disrupt the economy of the country, causing infrastructure damage, injury and the loss of life. In this situation, it might make sense to provide an intelligent computing system to perform automated interpretation of satellite images, especially identifying cloud cover region. The organization of the paper is as follows: Section 2 explains the Speeded Up Robust Features to obtain the affine corrected images. Cloud regions are segmented from the affine corrected test image by Otsu thresholding followed by latitude and longitude identification. Section 3 depicts results and discussion in which the proposed method is compared with an existing Adaptive Average Brightness Thresholding technique for cloud segmentation and finally Section 4 provides conclusion and future directions.

\section{MethodologY}

Cloud plays a vital role in weather forecasting. The use of cloud cover in weather prediction has led to various weather lore over the centuries. The overview of an automated image processing method based on cloud segmentation is shown in Fig.1. Satellites for meteorological purposes are used to acquire a sequence of images of earth. At the time of every acquisition, the way the satellite focuses the earth differs in terms of scale, translation and rotation and these images are taken as test image for further analysis. A less cloud covered satellite image is taken as a reference image. The transformation between the reference and test image is calculated using the descriptors obtained from speeded up robust features, The parameters of transformation are applied on the test image to obtain the affine corrected test image which is similar in scale space to the reference image. Cloud regions are segmented from the affine corrected images based on Otsu thresholding. Grids are overlayed on the segmented image to estimate the latitude and longitude of the cloud covered region. 


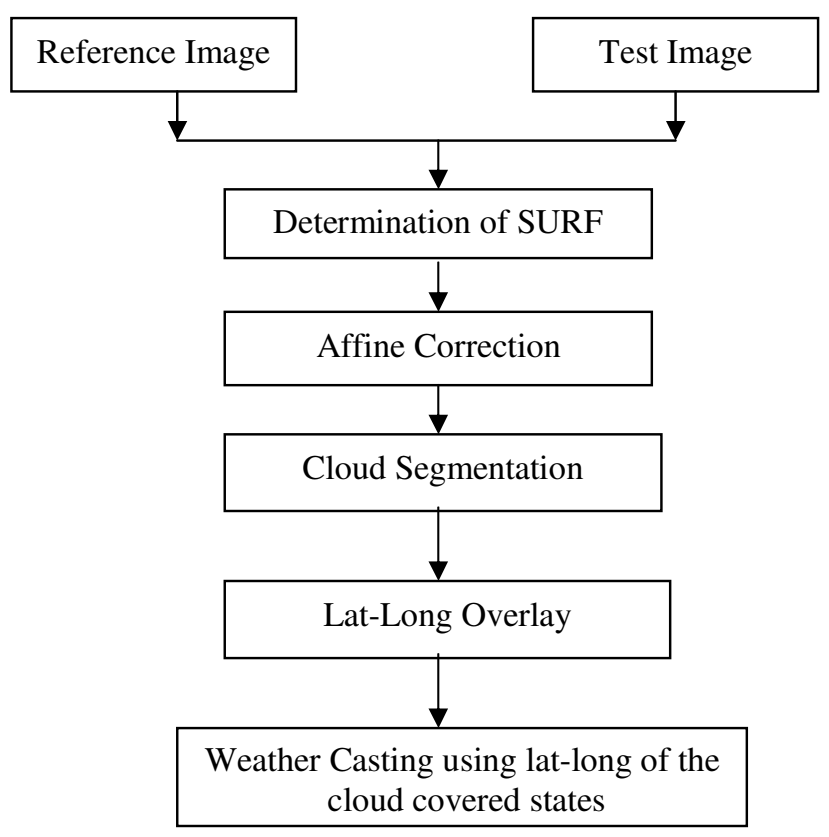

Fig 1: Overview of proposed Algorithm

\subsection{Feature Extraction}

The feature extraction method used in the proposed paper is Speeded Up Robust Features. The features so obtained are robust in the sense that they are invariant to translation, rotation, scale and affine transforms. The feature extraction method used in the proposed paper is Speeded Up Robust Features (SURF). The SURF algorithm consists of following modules namely

\subsubsection{Integral Images}

Much of the performance increase in SURF can be attributed to the use of an intermediate image representation known as the Integral Image. The integral image is computed rapidly from an input image and is used to speeded up the calculation of any upright rectangular area. Given an input image I and a point $(x ; y)$ the integral image IP is calculated by the sum of the values between the point and the origin. Formally this can be defined by the formula

$$
I_{\Sigma}(x, y)=\sum_{i=0}^{i \leq x} \sum_{j=0}^{j \leq y} I(x, y)
$$

Since computation time is invariant to change in size this approach is particularly useful when large areas are required. SURF makes good use of this property to perform fast convolutions of varying size box filters at near constant time.

\section{1) Fast Hessian Detector}

The first step in the Fast-Hessian process is to construct the blob-response map for the desired number of octaves and intervals. The default settings of three octaves with four intervals each are 
constructed as the scale space. The values for each octave-interval pair dictate the size of the filter which is convolved at that layer in the scale-space. The filter size is given by the formula,

$$
\text { Filter Size }=3\left(\left(2^{\circ} \times i\right)+1\right)
$$

Where ' $o$ ' is the octave and ' $i$ ' is the interval. Bay proposed an approximation to the Laplacian of Gaussians by using box filter representations of the respective kernels which is shown in Fig.2. For the Dxy filter the black regions are weighted with a value of -1 , the white regions with a value of 1 and the remaining areas not weighted at all. The Dxx and Dyy filters are weighted similarly but with the white regions have a value of 1 and the black with a value of -2 . Considerable performance increase is found when these filters are used in conjunction with the integral image.

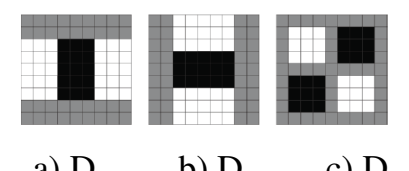

Fig 2: Weighted Box filters Approximation of LOG

In order to detect interest points using the determinant of Hessian it is first necessary to introduce the notion of a scale-space. A scale-space is a continuous function which can be used to find extrema across all possible scales. In computer vision the scale-space is typically implemented as an image pyramid where the input image is iteratively convolved with Gaussian kernel and repeatedly sub-sampled. As the processing time of the kernels used in SURF is size invariant, the scale-space can be created by applying kernels of increasing size to the original image. This allows for multiple layers of the scale-space pyramid to be processed simultaneously and negates the need to sub sample the image hence providing performance increase.

Once the responses are calculated for each layer of the scale-space, it is important that they are scale-normalized. Scale-normalization is an important step which ensures there is no reduction in the filter responses as the underlying Gaussian scale increases. For the approximated filters, scalenormalization is achieved by dividing the response by the area. This yields near perfect scale invariance. The search for extrema in the response map is conducted by a non-maximal suppression as shown in Fig. 4. Each pixel is compared to its 26 neighbors of adjacent scale and only those which are greater than all those surrounding it are classified as interest points.

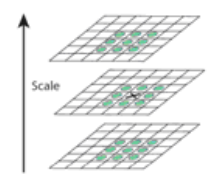

Fig 4: Extrema Detection

\section{2) Interest Point Descriptor}

The SURF descriptor describes how the pixel intensities are distributed within a scale dependent neighborhood of each interest point detected by the Fast-Hessian. Haar wavelets are used in order to increase robustness and decrease computation time. Haar wavelets are simple filters which can be used to find gradients in the $\mathrm{x}$ and $\mathrm{y}$ directions. Extraction of the descriptor can be divided into two distinct tasks. First each interest point is assigned a reproducible orientation before a scale dependent window is constructed in which a 64-dimensional vector is extracted. In order to 
achieve invariance to image rotation each detected interest point is assigned a reproducible orientation. Extraction of the descriptor components is performed relative to this direction so it is important that this direction is found to be repeatable under varying conditions.

To determine the orientation, Haar wavelet responses of size $4 \sigma$ are calculated for set pixels within a radius of $6 \sigma$ of the detected point, where $\sigma$ refers to the scale at which the point was detected. The specific set of pixels is determined by sampling those from within the circle using a step size of $\sigma$. The responses are weighted with a Gaussian centered at the interest point. Gaussian is dependent on the scale of the point and chosen to have standard deviation $2.5 \sigma$. Once weighted the responses are represented as points in vector space, with the x-responses along the abscissa and the y-responses along the ordinate. The dominant orientation is selected by rotating a circle segment covering an angle of $\pi / 3$ around the origin. At each position, the $\mathrm{x}$ and $\mathrm{y}$-responses within the segment are summed and used to form a new vector. The longest vector lends its orientation the interest point.

SURF descriptor is obtained by constructing a square window around the interest point. This window contains the pixels which will form entries in the descriptor vector and is of size $20 \sigma$, again where $\sigma$ refers to the detected scale. Furthermore the window is oriented along its dominant direction as mentioned previously. The descriptor window is divided into 4 x 4 regular sub regions. Within each of these subregions, Haar wavelets of size $2 \sigma$ are calculated for 25 regularly distributed sample points. Then, the wavelet responses $d x$ and $d y$ are summed up over each sub region and form a first set of entries to the feature vector.

In order to bring in information about the polarity of the intensity changes, the sum of the absolute values of the responses, $|\mathrm{dx}|$ and $|\mathrm{dy}|$ are extracted. Hence, each sub-region has a fourdimensional descriptor vector $\mathrm{v}$ for its underlying intensity structure as expressed in equation 3 . This results in a descriptor vector for all $4 \times 4$ sub-regions of length 64 . These 64 descriptors of each key point of the query and reference image is used for feature matching.

$$
V=\left(\sum d x, \sum d y, \sum|d x|, \sum|d y|\right)
$$

\section{3) Correlation over scale space}

Due to the change in satellite view point on earth, the scale of the test image varies from that of the reference image. Hence, these raise a need for correlation between the correct scale of test and reference image.

The scale space generated in SURF for locating feature points is used here. The scale in scale space increases such that the top most layer the highest scale and the bottom layer has the least scale. Considering that there are ' $\mathrm{m}$ ' numbers of matched features obtained through the Descriptor ratio method.

With available matched point, correlation method is to be applied only between the corresponding matches in query image. The error is computed by considering the neighbourhood pixels of the feature point and the neighbourhood pixels of the feature point its corresponding match in a single scale of the test image. This process continues until the errors for the same feature point in

highest scale of the image is computed. Then the pair scale that produces the minimum error for the considered feature point is detected. Then the entire process is repeated for all the ' $\mathrm{m}$ ' pair of matched points. Error ' $\mathrm{e}$ ' is calculated for all the points in all the scale pairs of ' $\mathrm{k}$ ' and ' $\mathrm{l}$ ' as given in expression. 
$e(i(k, l))=\left\{\frac{d(k, l)}{(2 r+1)^{2}}\right\}$

where

$$
\begin{aligned}
& d(k, l)=\sum_{u=-r v=-r}^{r} \sum^{r} Q(\alpha, \beta)-R(\alpha, \beta), \\
& \alpha=x_{i}+u, \beta=y_{i}+v
\end{aligned}
$$

For $i=1,2 \ldots . M, k=1,2, \ldots N, l=1,2, \ldots N$. . $N$ ' is the number of scales in scale space of Query image and reference image and ' $M$ ' is the number of matched feature points obtained through the descriptor ratio method. For each matched feature point, find ' $k$ ' and ' 1 ' such that $e(i(k, l)$ ) is minimum. Let ' $C s Q$ ' and ' $C s R$ ' be the closest scales of Query image and reference image. After finding the closest pair of scale, the mean of errors (merr) is computed for all the feature point pair in the closest scale pair as per the equation 1

$$
\operatorname{merr}=\left\{\frac{\sum_{i=1}^{M} e(i(k=C s Q, \quad I=C s R))}{i}\right\}
$$

Only the true feature points that are located in common field of view of both test and reference image gets matched. Those points that are located outside the overlapping regions are classified as mismatches and are rejected for further processing. 


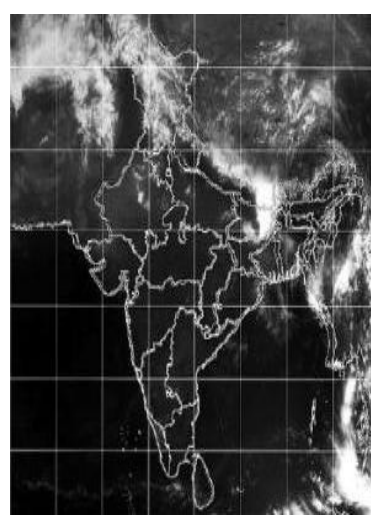

Fig 4: Reference image

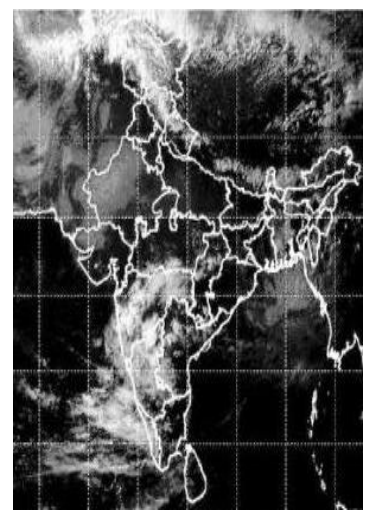

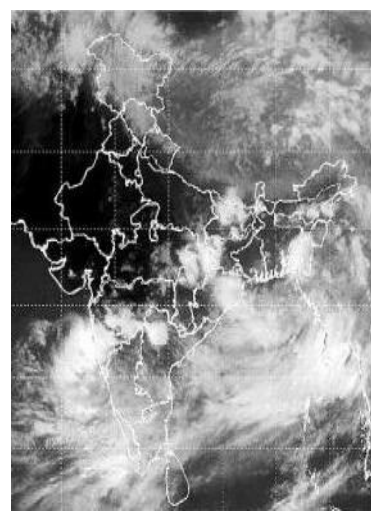

Fig 5: Test Image 1

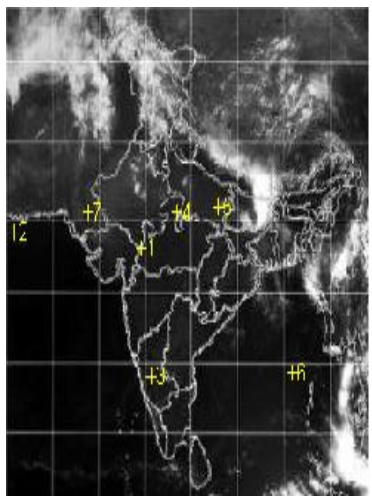

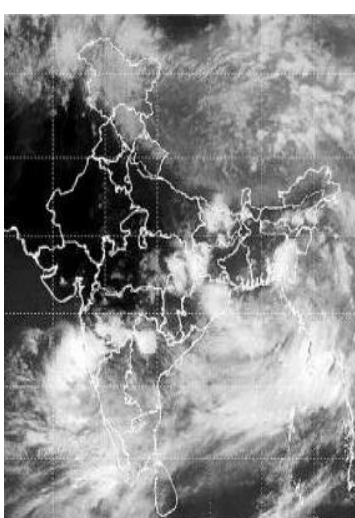

Fig 6: Test

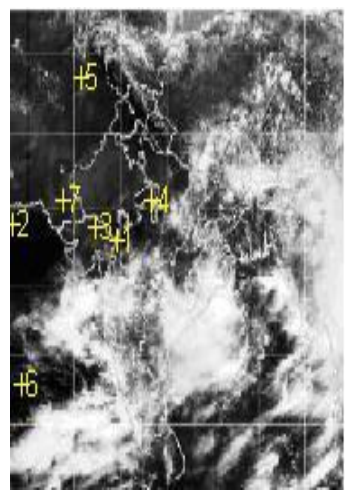

Fig 7: Test image 3 Fig 8: Descriptors of Reference Fig 9: Descriptors of Test Image1

\subsection{Affine Transformation}

The transformation parameters are calculated from the final matched points in the reference and test image using the affine transformation matrix given below.

$$
\left[\begin{array}{cc}
s^{*} \cos \theta & -s^{*} \sin \theta \\
-s^{*} \sin \theta & s * \cos \theta \\
t x & t y
\end{array}\right]
$$

where's' denotes scaling, refers to orientation and 'tx', 'ty' are the translation along $\mathrm{x}$ and $\mathrm{y}$ direction respectively. The affine parameters calculated from the matched feature points is used to correct the test image to make the scaling and orientation same as the reference image. 


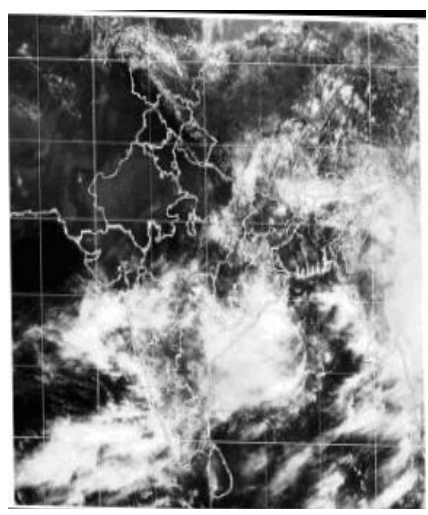

Fig 10: Affine Corrected Test Image1

\subsection{Thresholding}

Otsu thresholding technique [9] has been used to segment cloud regions from the input image. As, many of the thresholding techniques depend on the shape of the histogram, Otsu thrsholding works stable without any apriori knowledge. Otsu's method of image segmentation selects an optimum threshold by maximizing the between-class variance in a gray image. An image is a $2 \mathrm{D}$ grayscale intensity function, and contains $\mathrm{N}$ pixels with gray levels from 1 to ' $L$ '. The number of pixels with gray level $\mathrm{i}$ is denoted fi, giving a probability of gray level $\mathrm{i}$ in an image of $\mathrm{pi}=\mathrm{fi} / \mathrm{N}$. In the case of bi-level thresholding of an image, the pixels are divided into two classes, ' $\mathrm{Cl}$ ' with gray levels $[1 \ldots \mathrm{t}]$ and ' $C 2^{\prime}$ with gray levels $[\mathrm{t}+1 \ldots \mathrm{L}]$. Then, the gray level probability distributions for the two classes are provided in equation (14)

$C_{1}: p_{1} / \omega_{1}(t), \ldots, p_{t} / \omega_{1}(t)$ and

$C_{2}: p_{t+1} / \omega_{2}(t), p_{t+2} / \omega_{2}(t), \ldots ., p_{L} / \omega_{2}(t)$

where $\omega_{1}(t)=\sum_{i=1}^{t} p_{i}$ also, the means for classes $C_{1}$ and $C_{2}$ are given in equation (16)

$\mu_{1}=\sum_{i=1}^{t} i p_{i} / \omega_{1}(t)$

$\mu_{2}=\sum_{i=t+1}^{L} i p_{i} / \omega_{2}(t)$

Let ' $\mu_{T}$ ' be the mean intensity for the whole image. It is easy to show that,

$$
\begin{aligned}
& \omega_{1} \mu_{1}+\omega_{2} \mu_{2}=\mu_{T} \\
& \omega_{1}+\omega_{2}=1
\end{aligned}
$$


Using discriminant analysis, Otsu defined the between-class variance of the thresholded image as given in (11)

$\sigma_{B}^{2}=\omega_{1}\left(\mu_{1}-\mu_{T}\right)^{2}+\omega_{2}\left(\mu_{2}-\mu_{T}\right)^{2}$

For bi-level thresholding, Otsu verified that the $t^{*}$ is chosen so that the between-class variance

$\sigma_{B}^{2}$ is maximized; that is given in equation (12),

$t^{*}=\operatorname{Arg} \operatorname{Max}\left\{\sigma_{B}^{2}(t)\right\} \quad 1 \leq t<L$

Let ' $X_{t}$ ' be the adaptive threshold based on Otsu method of the input image ' $X$ ' and assume that $X_{t} \in\left\{X_{0}, X_{1}, \ldots, X_{L-1}\right\}$. Designate the first subimage as ' $X_{s 1}{ }^{\prime}$ consisting of gray levels $\left\{\mathrm{X}_{\mathrm{o}}, \mathrm{X} 1 \ldots \mathrm{X}_{\mathrm{t}}\right\}$, subimage $X_{s 2}{ }^{\prime}$ consisting of gray levels $\left\{\mathrm{X}_{\mathrm{t}+1} \ldots \mathrm{X}_{\mathrm{L}-1}\right\}$. The adaptive threshold obtained from equation (12) can be used to extract the foreground and background image pixels. Because of the global equalization, the distortion of visual quality in the background is retrieved with the original input pixels. The enhanced object features are retained as such in the enhanced image. Threshold value is selected to segment cloud region by maximizing the criterion function and these segmented regions are labeled based on its connectivity.

\subsection{Connected Component Analysis}

Cloud region is determined by means of a region based operation. The segmented cloud region is scanned pixel by pixel in order to identify connected pixel regions where each pixel is denoted as 'V', i.e. regions of adjacent pixels which share the same set of intensity values. Connected component analysis with 8-connectivity, scans the image by moving along a row until it comes to a point $\mathrm{p}$ (where $\mathrm{p}$ denotes the pixel to be labelled at any stage in the scanning process) for which $V=\{1\}$. When this is true, it examines the four neighbours of $p$, which have already been encountered in the scan. After completing the scan, the equivalent label pairs are sorted into equivalence classes and a unique label is assigned to each class. As a final step, a second scan is made through the image, during which each label is replaced by the label assigned to its equivalence classes. Thus the labels will represent the distinct regions of the segmented image.

\subsection{Weak Boundary Elimination}

Let $\mathrm{R}=\mathrm{R} 1, \mathrm{R} 2, \ldots ., \mathrm{Rn}$ be the set of labelled regions. Let ' $\mathrm{M}$ ' and ' $\mathrm{N}$ ' denote the rows and columns of each region. The number of pixels representing each region $\mathrm{Rk}$ is given by

$\mathrm{NP}=\mathrm{Rk}(\mathrm{i}, \mathrm{j})$

As the images are acquired in a non-ideal environment there is a need to remove non cloud regions, caused by interference from the aircraft systems, complex terrain including snow, cover, land and ice. Continuous and massive cloud concentrated over a particular region is identified as true cloud. In this work, Regions with area lesser than 20 percent of the maximum number of pixels are considered as noise and interference due to cluttered environment. The latitude and longitude of the cloud covered region has to be estimated to locate the region geographically. 


\subsection{Lat-Long Identification}

Latitude and longitude are the horizontal and vertical mapping lines of the earth. The latitude of India ranges from $7^{\circ} 30^{\prime} \mathrm{N}$ to $35^{\circ} \mathrm{N}$ and longitude from $65^{\circ} 66^{\prime} \mathrm{E}$ to $97^{\circ} 30^{\prime} \mathrm{E}$. Cloud covered regions are identified by forming the grid over the segmented image. The Universal Transverse Mercator Coordinate (UTM) system provides coordinates on a world wide flat grid for easy computation which divides the world in to 60 zones [17]. In this work, Indian Territory is subdivided in to 256 zones by forming $16 \times 16$ grids. Latitude and longitude of each cloud covered region are estimated approximately from the grid numbers as shown in Table 1 . The block $(1,1)$ represents the leftmost top corner of the image and is traversed in horizontal direction and then in vertical direction.

\section{Table 1}

Representative Look Up Table For Latitude And Longitude Identification

\begin{tabular}{|c|c|c|c|}
\hline $\begin{array}{l}\text { Block } \\
\text { No }\end{array}$ & Latitude & Longitude & States covered \\
\hline$(1,1)$ & $\begin{array}{l}35^{\circ} 28^{\prime \prime}-7^{\circ} \\
30^{\prime \prime}\end{array}$ & $65^{\circ} 66-73^{\circ} 57^{\prime}$ & Out of Boundary \\
\hline$(1,2)$ & $\begin{array}{l}35^{\circ} 28^{\prime \prime}-7^{\circ} \\
30^{\prime \prime}\end{array}$ & $73^{\circ} 57^{\prime}-81^{\circ} 48^{\prime}$ & $\begin{array}{l}\text { Chandigarh,Haryana ,Himachal Pradesh } \\
\text { Jammu and KashPunjab,Uttarkhan }\end{array}$ \\
\hline$(1,3)$ & $\begin{array}{l}35^{\circ} 28^{\prime \prime}-7^{\circ} \\
30^{\prime \prime}\end{array}$ & $81^{\circ} 48^{\prime}-89^{\circ} 39^{\prime}$ & Out of Boundary \\
\hline$(1,4)$ & $\begin{array}{l}35^{\circ} 28^{\prime \prime}-7^{\circ} \\
30^{\prime \prime}\end{array}$ & $89^{\circ} 39^{\prime}-97^{\circ} 30^{\prime}$ & Out of Boundary \\
\hline$(2,1)$ & $\begin{array}{l}28^{\circ} 70^{\prime \prime}-21 \\
15^{\prime}\end{array}$ & $\begin{array}{l}65^{\circ} 66-73^{\circ} \\
57^{\prime}\end{array}$ & Gujarat \\
\hline$(2,2)$ & $\begin{array}{l}28^{\circ} 7^{\prime} 30^{\prime \prime}-21 \\
15^{\prime}\end{array}$ & $73^{\circ} 57^{\prime}-81^{\circ} 48^{\prime}$ & $\begin{array}{l}\text { Delhi, Goa,Madya pradesh,Rajasthan } \\
\text { Uttar Pradesh }\end{array}$ \\
\hline$(2,3)$ & $\begin{array}{l}28^{\circ} 70^{\prime \prime}-21 \\
15^{\prime}\end{array}$ & $81^{\circ} 48^{\prime}-89^{\circ} 39^{\prime}$ & $\begin{array}{l}\text { Bihar,Chattisgrah,Jharkhand,Sikkim } \\
\text { West Bengal }\end{array}$ \\
\hline$(2,4)$ & $\begin{array}{l}28^{\circ} 7^{\prime} 30^{\prime \prime}-21 \\
15^{\prime}\end{array}$ & $89^{\circ} 39^{\prime}-97^{\circ} 30^{\prime}$ & $\begin{array}{l}\text { Arunachal Pradesh,Assam,Manipur } \\
\text { Meghalaya,Mizoram,Nagaland,Tripura }\end{array}$ \\
\hline$(3,1)$ & $\begin{array}{l}21^{\circ} 15^{\prime}-14^{\circ} \\
22^{\prime} 30^{\prime \prime}\end{array}$ & $\begin{array}{l}65^{\circ} 66-73^{\circ} \\
57^{\prime}\end{array}$ & Daman and Diu \\
\hline$(3,2)$ & $\begin{array}{l}21^{\circ} 15^{\prime}-14^{\circ} \\
22^{\prime} 30^{\prime \prime}\end{array}$ & $73^{\circ} 57^{\prime}-81^{\circ} 48^{\prime}$ & Andhra pradesh,Karnataka,Maharashtra \\
\hline$(3,3)$ & $\begin{array}{l}21^{\circ} 15^{\prime}-14^{\circ} \\
22^{\prime} 30^{\prime \prime}\end{array}$ & $81^{\circ} 48^{\prime}-89^{\circ} 39^{\prime}$ & Orissa \\
\hline$(3,4)$ & $\begin{array}{l}21^{\circ} 15^{\prime}-14^{\circ} \\
22^{\prime} 30^{\prime \prime}\end{array}$ & $89^{\circ} 39^{\prime}-97^{\circ} 30^{\prime}$ & Out of Boundary \\
\hline$(4,1)$ & $\begin{array}{l}14^{\circ} 22^{\prime} 30^{\prime \prime}-7 \\
\text { o } 30^{\prime}\end{array}$ & $65^{\circ} 66-73^{\circ} 57^{\prime}$ & Lakshadweep \\
\hline$(4,2)$ & $\begin{array}{l}14^{\circ} 22^{\prime} 30^{\prime \prime}-7 \\
\text { o } 30^{\prime}\end{array}$ & $73^{\circ} 57^{\prime}-81^{\circ} 48^{\prime}$ & Kerala,Tamil Nadu, $\square \square$ Pondicherry \\
\hline$(4,3)$ & $\begin{array}{l}14^{\circ} 22^{\prime} 30^{\prime \prime}-7 \\
\text { o } 30^{\prime}\end{array}$ & $81^{\circ} 48^{\prime}-89^{\circ} 39^{\prime}$ & Out of Boundary \\
\hline$(4,4)$ & $\begin{array}{l}14^{\circ} 22^{\prime} 30^{\prime \prime}-7 \\
{\text { o } 30^{\prime}}\end{array}$ & $89^{\circ} 39^{\prime}-97^{\circ} 30^{\prime}$ & Andaman and Nicobar \\
\hline
\end{tabular}

\section{RESULTS AND DISCUSSION}

To demonstrate the efficacy of the proposed technique, 60 sample images with a spatial resolution of $1 \mathrm{Km}$ captured by KALPANA-1 satellite in infrared band were taken from the website of Indian Meteorological Department . SIFT is slow and not good at illumination changes, while it is invariant to rotation, scale changes and affine transformations SURF shows its stability and fast 
speed in the experiments. It is known that 'Fast-Hessian' detector that used in SURF is more than 3 times faster that DOG (which was used in SIFT) and 5 times faster than Hessian-Laplace.The image with least cloud covered region from the collected dataset is chosen as the reference image which is shown in Fig.5 while the test image with different scale is shown in Fig.6-8. Keypoint descriptors are found for reference and test image by varying the threshold value in the range 0.1 to 0.9 , typically 0.75 and they are shown in Fig.8 and Fig.9. This is followed by the calculation of transformation parameters for matched feature points which are applied to the test image as in Fig.6-8.

The proposed method is compared with the existing Adaptive Average Brightness Thresholding technique [8]. In this method, the average brightness of the digitized, grayscale image is calculated. Next, a threshold brightness value is chosen based on the average brightness of the image. Finally, this threshold is applied to the image to divide it into different regions of brightness. The first decision is to decide on the threshold brightness level. Weather satellite images can range from ones with no cloud, over bodies of water, to ones with almost complete cloud coverage. These two cases would provide extremely dark and extremely bright levels of average brightness, respectively. Adaptive Average Brightness Thresholding uses an averagecutoff function to determine the appropriate brightness threshold level. This function has the characteristic that at low average brightness levels, the cutoff is very much above the average, whereas at high average brightness levels, the cutoff is only marginally above the average brightness.

$\left.\operatorname{cutoff}(1)=\sum_{i=k+1}^{k+r / 2 m+c / 2} \sum_{j=m+!} i m(i, j)+f *(\ln (G \max ))-\ln \left(\sum_{i=k+1}^{k+r / 2 m+c / 2} \sum_{j=m+1}^{2} i m(i, j)\right)\right)$

where 'im' refers to the affine corrected test image, ' $r$ ' and ' $c$ ' refers to the size of the image, ln denotes the natural logarithm, 'Gmax' is the number of grayscale values, ' $\mathrm{f}$ ' is the multiplicative coefficient, determined empirically. The parameters ' $\mathrm{k}$ ' and ' $\mathrm{m}$ ' are used to navigate across the four quadrants of the image.

The cutoff functions for the four quadrants of the test image using Adaptive Average Brightness Thresholding are 148.3643, 158.7722, 93.3247, and 109.8715. The cloud image is segmented from the original image using the cutoff function and the segmented cloud image by eliminating the weak boundaries is shown in Fig.16.The results depict that the segmented cloud image obtained from the proposed technique is better than the Adaptive Average Brightness Algorithm. The latitude and longitude of the cloud covered region is estimated by superimposing $16 \times 16$ grids over the original image. The states that are covered by cloud is found by using the look up table. Pseudo coloring is applied to the resultant segmented images from Otsu and AABT as shown in Fig.17 -Fig.18and Fig.19-Fig.20. The segmentation of NOAA satellite image was performed using OTSU and AABT algorithm. The NOAA satellite image is shown in Fig 21 . The satellite images are not ideal because of noise interference from the aircraft systems ,complex terrain including land,snow cover,ice and snow pixels with similar brightness values.Clouds segmented are shown in green colour and undetected clouds are in red color. The results depict that the cloud region was better segmented using Otsu thresholding algorithm rather than Adaptive Average Brightness Algorithm. Hence segmentation by OTSU thresholding is chosen to identify cloud cover regions. The proposed algorithm has been tested with different sets of images from website of Indian Meteorological Department from June, 2011 to July, 2011 and the results are shown in Fig 11-20 and the cloud covered states are tabulated in Table 2. 


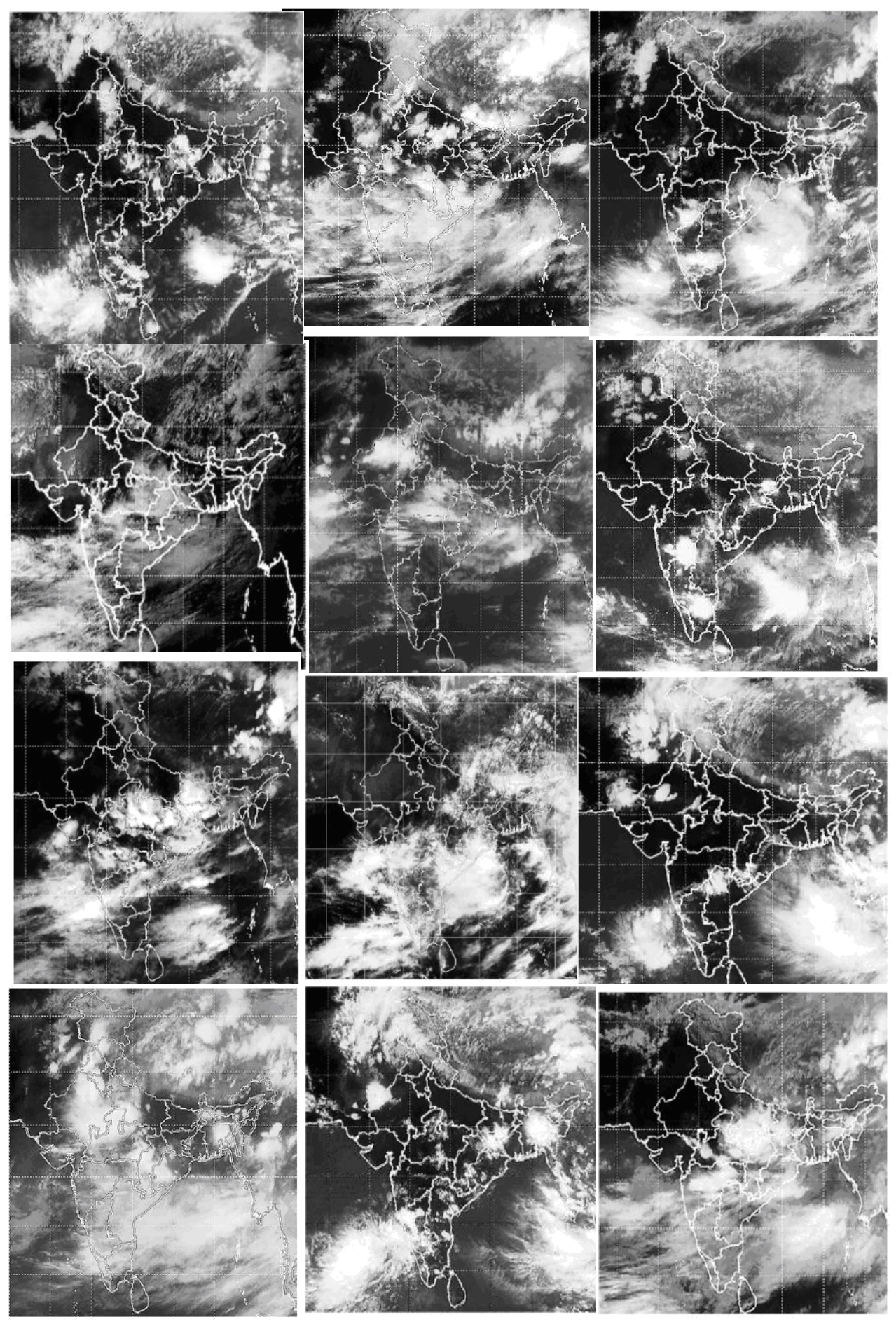

Fig .11 Database of cloud covered images 


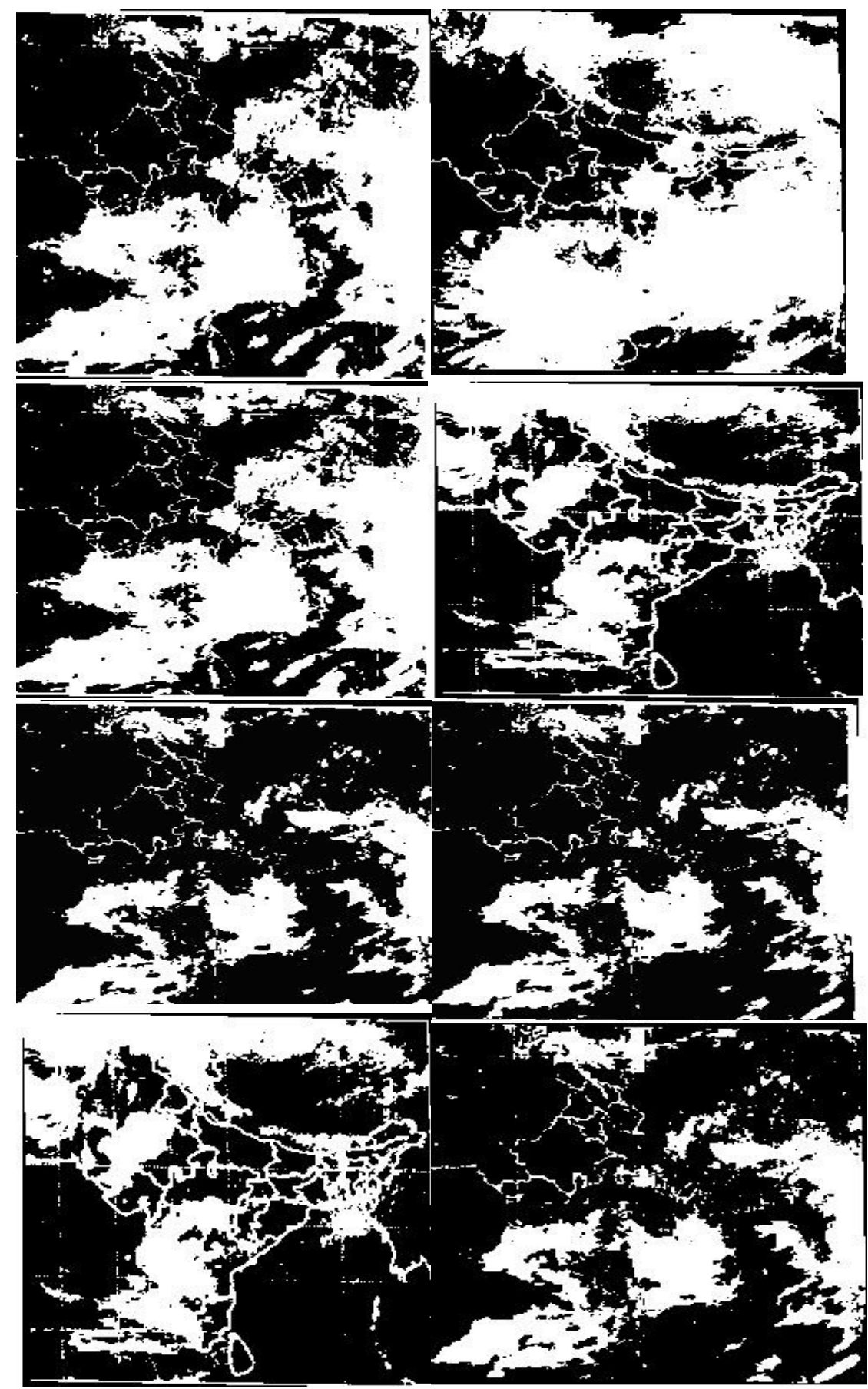

Fig .12 Affine Corrected And Otsu Segmented Images 
Table 2

Cloud Covered States

\begin{tabular}{|c|c|c|c|}
\hline $\begin{array}{c}\text { Test } \\
\text { image }\end{array}$ & Latitude & Longitude & States covered \\
\hline \multirow[t]{9}{*}{ Fig.5 } & $30^{\circ} 42$ & $76^{\circ} 54^{\prime}$ & Chandigarh \\
\hline & $30^{\circ} 15^{\prime}$ & $79^{\circ} 15^{\prime}$ & Uttar Khand \\
\hline & $28^{\circ}$ & $95^{\circ}$ & Arunachal Pradesh \\
\hline & $27^{\circ} 30^{\prime}$ & $88^{\circ} 3^{\prime}$ & Sikkim \\
\hline & $15^{\circ} 7^{\prime}$ & $75^{\circ} 2^{\prime}$ & Karnataka \\
\hline & $25^{\circ} 30^{\prime}$ & $91^{\circ} 1^{\prime}$ & Meghalaya \\
\hline & $29^{\circ} 8^{\prime}$ & $76^{\circ} 5^{\prime}$ & Delhi \\
\hline & $21^{\circ}$ & $75^{\circ}$ & Maharashtra \\
\hline & $9^{\circ} 7^{\prime}$ & $76^{\circ} 5$ & Kerala \\
\hline \multirow[t]{8}{*}{ Fig.6 } & $30^{\circ} 42^{\prime}$ & $76^{\circ} 54$ & Chandigarh \\
\hline & $27^{\circ} 30^{\prime}$ & $88^{\circ} 3^{\prime}$ & Sikkim \\
\hline & $28^{\circ}$ & $95^{\circ}$ & Arunachal Pradesh \\
\hline & $29^{\circ} 85$ & $75^{\circ} 85^{\prime}$ & Punjab \\
\hline & $15^{\circ}$ & $75^{\circ}$ & Karnataka \\
\hline & $16^{\circ}$ & $80^{\circ}$ & Andhra Pradesh \\
\hline & $25^{\circ} 30^{\prime}$ & $91^{\circ}$ & Meghalaya \\
\hline & $11^{\circ}$ & $78^{\circ}$ & Tamil Nadu \\
\hline \multirow[t]{5}{*}{ Fig.7 } & $11^{\circ}$ & $78^{\circ}$ & Delhi \\
\hline & $30^{\circ} 5$ & $74^{\circ} 9^{\prime}$ & Jammu and Kashmir \\
\hline & $20^{\circ} 8^{\prime}$ & $75^{\circ}$ & Maharashtra \\
\hline & $15^{\circ}$ & $75^{\circ}$ & Karnataka \\
\hline & $9^{\circ} 7^{\prime}$ & $76^{\circ} 5$ & Kerala \\
\hline
\end{tabular}

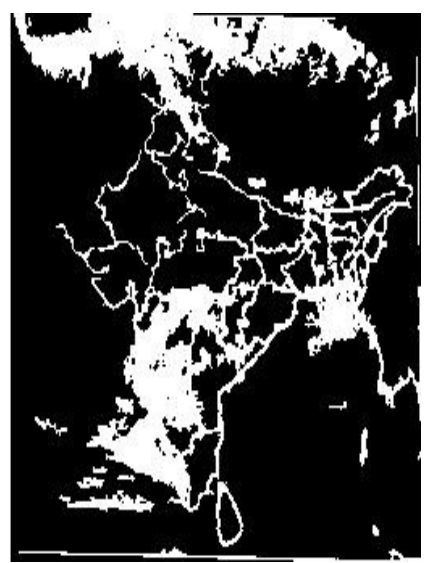

Fig 13: AABT Segmented Image 1

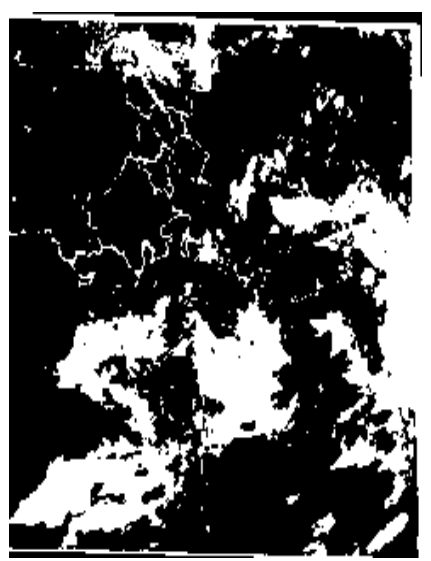

Fig14: AABT Weak Boundary Elimination 


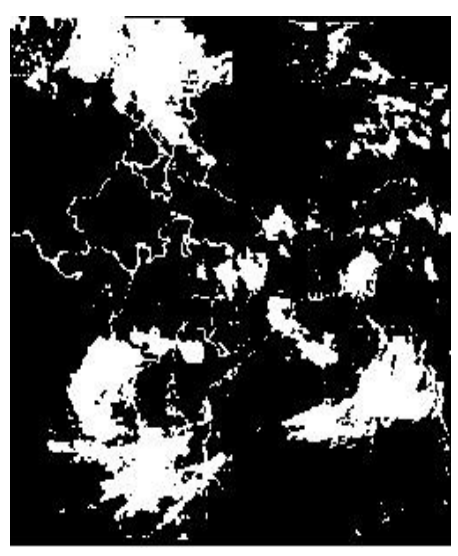

Fig 15: AABT Segmented image2

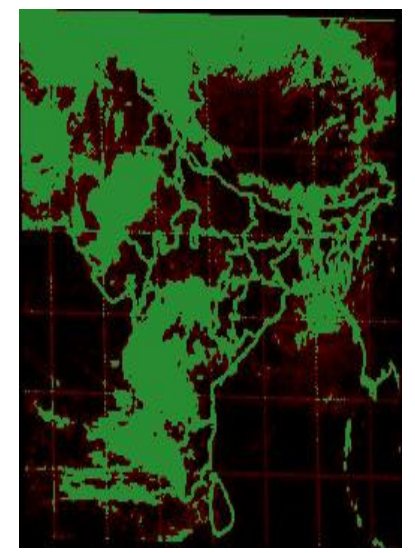

Fig17: Pseudo color map of

Otsu segmented image3

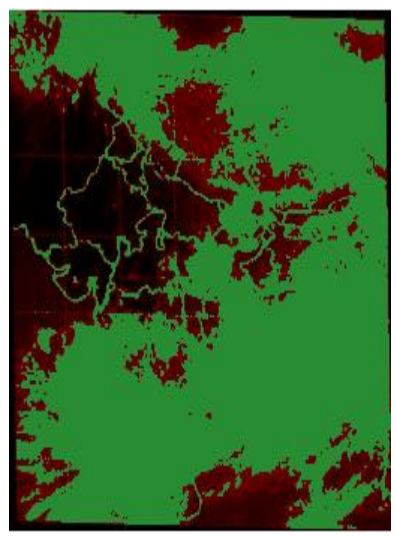

Fig19: Pseudo color map of

Otsu segmented image2

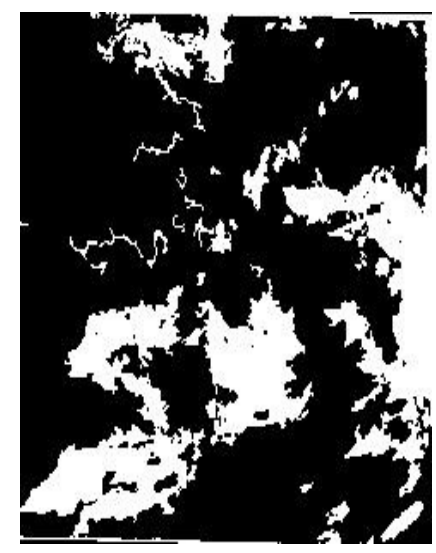

Fig16: AABT Weak

Boundary elimination

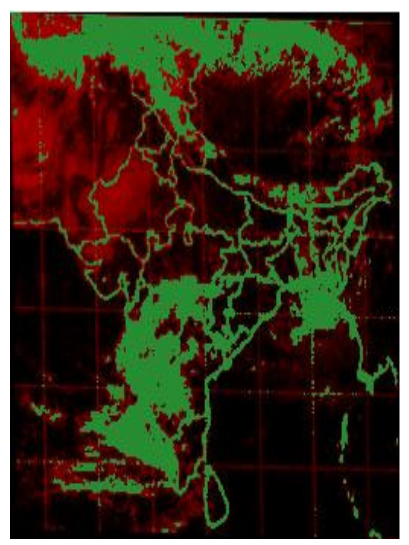

Fig18: Pseudo color map of AABT segmented image3

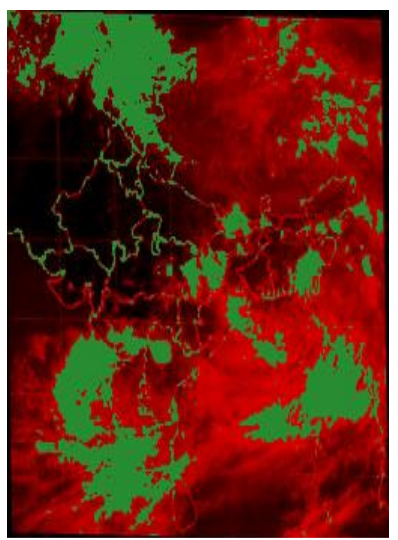

Fig20: Pseudo color map of AABT segmented image2 


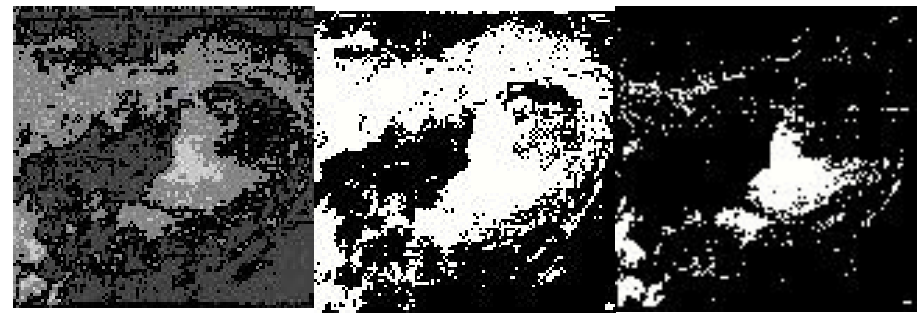

Fig 21. NOAA Image Otsu Segmented AABT Segmented

\section{CONCLuSion}

In this paper, a method for automatic weather forecasting by segmenting cloud images irrespective of scale, translation and rotations is proposed. Speeded Up Robust Feature Transform (SURF) is incorporated in this work to bring the captured satellite image similar to the reference image. The cloud regions are segmented using the Otsu thresholding followed by connected component analysis. Latitude and longitude positions were identified by forming a grid over the segmented image and the cloud covered states are identified. This algorithm has been tested over INSAT images and compared against Adaptive Average Brightness Thresholding (AABT).The proposed method outperforms the existing AABT. This work can be extended to explicitly identify the cloud cover area specific to districts by increasing the number of lat-long grids and also to cloud tracking by estimating the cloud motion vectors for live weather casting.

\section{REFERENCES}

[[1] M.Imran,K.M.Riaz,A.Ajith INeuro-Computing based Canadian weather analysis," International workshop on Intelligent Systems design and applications., vol. 6, pp.39-44,2002, in press.

[2] C.A.Doswell, Short range forecasting: Mesoscale meterology and forecasting," Journal of Applied Meterology., chap.29,pp. 689-719,1986, in press.

[3] A.Ajith,P.Sajith and J.Babu, IWill We Have a Wet Summer Long-term Rain Forecasting Using Soft Computing Models," 15th European Simulation Multi Conference , pp.1044-1048,2001, in press.

[4] Kuligowski R. J., Barros A. P , Localized precipitation forecasts from a numerical weather prediction model using arti_cial neural networks," Journal of AppliedMeterologyl. Vol.13, pp. 1194-1204, 1998, in press.

[5] Sin Liang Lim ,B. S. Daya Sagar"Cloud field segmentation via multiscale convexity analysis" ,JOURNAL OF GEOPHYSICAL RESEARCH, VOL. 113, D13208, 17 PP., 2008, in press.

[6] G. S. Pankiewicz., IPattern recognition techniques for identi_cation of cloud and cloud systems," Journal of Applied Meterology. vol. 2, pp.257-271,September 1995, in press.

[7] Khan Muhammad Riaz and Ondrusek Cestmir., IShort-term load forecasting with multilayer perceptron and recurrent neural network," Journal of ElectricalEngineering, vol.53, pp.17-23,February 2002 , in press.

[8] Isaac J.H. Leung, James E. Jordan, `Image Processing for Weather Satellite Cloud Segmentation," Electrical and Computer Engineering,Canadian Conference., vol.2, pp. 953-956,Sep 1995, in press.

[9] Ping-sung liao, Tse-sheng chen and Pau-choo chung, \A Fast Algorithm for Multilevel Thresholding," Journal of Information Science and Engineering, vol.17, pp713-727,2001, in press.

[10] J.Lee,R.Weger, IA neural network approach to cloud classi_cation:.,IEEE trans. Geoscience Remote sensing, vol.28, pp 846-855,1990, in press.

[11] J.Peak and P.Tag, ISegmentation of satellite imagery using hierarchial thresholding and neural networks," Journal of Applied Metero logy, vol.33,pp 605-616,1994, in press.

[12] B.Tian,A.Mukhtair,R.Mahmood,T.V Haar,LA study of cloud classi_cation with neural networks using spectral and textural features," IEEE Trans.NeuralNetworks, vol.10,pp138-151,1999, in press. 
[13] S.Yhann and J.Simpson,LApplication of neural networks to AVHRR cloud segmentation," IEEE Trans. Geoscience Remote Sensing, vol.33,pp 590-604, in press.

[14] Dipti Prasad Mukherjee,Scott T.Acon,ICloud Tracking by scale space classi_cation," IEEE Trans.Geoscience Remote Sensing, vol.40,pp 405-415, 2002, in press.

[15] David Lowe,"Distinctive Image Features from Scale-Invariant Keypoints," International Journal of Computer Vision, 2004,in press.

[16] David Lowe, "Object Recognition from Local Scale-Invariant Features," Proc. of the International Conference on Computer Vision., 1999, in press.

[17] Fei Wenlong Lv Hong Wei Zhihui "Satellite Cloud Image Segmentation Based on the Improved Normalized Cuts Model",Information Science and Engineering (ICISE), 2009 1st International Conference. 2009, in press.

[18] Moro Sancho Q. I., Alonso L. and Vivaracho C. E., ,Application of neural networks to weather forecasting with local data,"Applied Informatics,Vol 16,No 11,1984, in press.

[19] http://casanovasadventures.com/catalog/gps/p3034.htm.

\section{Authors}

S.Mohamed Mansoor Roomi received his B.E degree in Electronics and communication Engineering from Madurai Kamarajar University, in 1990.and the M. E(Power Systems)\&ME (Communication Systems) from Thiagarajar College of Engineering, Madurai in 1992\&1997 and $\mathrm{PhD}$ in 2009 from Madurai Kamarajar University. His primary Research Interests include Image Enhancement and Analysis.

Bhargavi is currently doing her under graduation in Electronics and Communication Engineering at Thiagarajar College of Engineering, Madurai,India. Her research interests are in the area of Image Enhancement and Image Analysis.

M.Hajira Rahima Banu is currently doing his under graduation in Electronics and Communication Engineering at Thiagarajar College of Engineering, Madurai,India.Her research interests are in the area of Image Enhancement and Image Analysis.
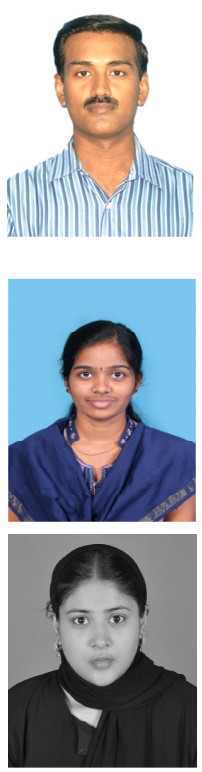